Zelmann R, Leijten FSS, Jeffreys JGR, Gotman J. High-frequency oscillations as a new biomarker for epilepsy. Ann Neurol February 2012;71:169-178). (Respond: Dr Zijlmans, Department of Neurology and Neurosurgery, University Medical Center Utrecht, The Netherlands. E-mail: g.j.m.zijlmans@umcutrecht.nl).

COMMENT. Methods used to determine the extent of epileptic foci of brain tissue before and during cortical resection include EEG telemetry, ECoG, MRI, histological, and immunohistochemical. Tissue markers of epileptic foci include mitochondrial "hypermetabolic" neurons and a-B-crystallin (Sarnat HB et al. Can J Neurol Sci 2011;38(6):909-17)(Sarnat HB, Flores-Sarnat L. ibid. 2009;36(5):566-74) (Pediatr Neurol Briefs 2012;26(1):5-6)(ibid. 2009;23(11);81-82). The Annals review provides a comprehensive account of the utility of HSOs as a biomarker of epileptogenesis.

HSOs are studied primarily in mesiotemporal epilepsies. In a series of 30 consecutive pediatric patients at UCLA undergoing surgery for refractory epilepsy due mainly to extratemporal lesions, ECoGs were recorded at $2,000 \mathrm{~Hz}$ and visually inspected for fast ripples (FR 250-500Hz). FR episodes were identified in ECoGs from 24 patients (80\%); FR-containing cortex was removed in 19 and all became seizure-free. FRcontaining cortex was found outside of abnormalities defined by MRI and FDG-PET in 6 children. The authors conclude that interictal fast ripples are an excellent surrogate marker of epileptogenesis. (Wu JY et al. Neurology 2010;75(19):1686-94).

\title{
EEG PATTERNS AND GENOTYPES IN ANGELMAN SYNDROME
}

Researchers at the Children's Hospital, Boston, other centers in the US, and Poznan University, Poland, prospectively analyzed EEGs from participants in the NIH Angelman Syndrome Natural History Study. Of 160 enrolled patients (2006-2010), 115 had complete data (58 boys, median age 3.6 years). EEG findings included intermittent rhythmic delta waves $(83.5 \%)$, interictal epileptiform discharges $(74.2 \%)$, intermittent rhythmic theta waves $(43.5 \%)$, and posterior rhythm slowing (43.5\%). Centro-occipital and centro-temporal delta waves decreased with age $(\mathrm{p}=0.01$ and 0.03$)$, and EEG patterns are age-dependent. EEG patterns and seizure types were not correlated significantly with genotypes. Using a classification tree to predict specific genotypes based on EEG features, deletions class-2 $(5.0 \mathrm{Mb})$ were associated with $>50 \%$ intermittent rhythmic theta activity, and deletions class-1 $(5.9 \mathrm{Mb})$ with $<50 \%$ intermittent rhythmic theta activity and epileptiform discharges while awake. EEG patterns are important biomarkers in Angelman syndrome and may suggest the underlying genetic etiology. (Vendrame M, Loddenkemper T, Zarowski M, et al. Analysis of EEG patterns and genotypes in patients with Angelman syndrome. Epilepsy Behav March 2012;23:261-265).(Response: Dr Sanjeev V Kothare, Children's Hospital Boston, Fegan 9, 300 Longwood Ave, Boston, MA 02115. E-mail: Sanjeev.Kothare@childrens.harvard.edu).

COMMENT. Boyd SG and colleagues at Great Ormond Street Children's Hospital, London, UK first described EEG patterns that were considered characteristic of Angelman syndrome: 1) Persistent rhythmic 4-6/s activity ( $>200 \mathrm{mcV})$ while awake; 2) prolonged runs of rhythmic $2-3 / \mathrm{sec}$ activity $(200-500 \mathrm{mcV})$ anteriorly; and 3) spikes 
mixed with $3-4 / \mathrm{s}$ waves $(>200 \mathrm{mcV})$ posteriorly, mainly with eye-closure. Discharges mixed with slow components on eye-closure was the most frequent finding in patients aged 11 months to $>12$ years. (Boyd SG et al. Eur J Pediatr 1988;147:508-513). Six children had no history of seizures and the EEG features helped identify patients at an early age.

The EEG findings in the present report are comparable to those of Boyd, and a notched delta pattern, also characteristic of Angelman syndrome, is found in patients presenting $<4$ years of age. Researchers at the Epilepsy Center, Children's Memorial Hospital, Chicago, evaluated the notched delta pattern in diagnosis of patients with a suggestive phenotype of Angelman syndrome. A retrospective review of video-EEG recordings with notched delta pattern found 38\% specificity for Angelman syndrome. (Korff CM, Kelley KR, Nordli DR. J Clin Neurophysiol 2005;22(4):238-243).

\section{INFECTIOUS DISEASES}

\section{EPILEPTOGENESIS IN CALCIFIED NEUROCYSTICERCOSIS}

Researchers at Sanjay Gandhi Post Graduate Institute of Medical Sciences, Lucknow, India, and University of Texas Medical School at Houston performed dynamic contrast-enhanced (DCE) MRI and determined serum matrix metalloproteinase-9 (MMP9) levels and MMP-9 gene polymorphisms in 30 subjects with a single calcified lesion of neurocysticercosis without any perilesional abnormality. These measures showed significant differences between 2 groups, each containing 15 patients, one with and one without seizures. In symptomatic subjects, serum MMP-9 levels and MMP-9 gene polymorphisms, the determinants of blood brain barrier permeability, were significantly higher compared with asymptomatic subjects with calcified cysticercus lesions and controls. There may be different degrees of perilesional inflammation with similar MRI calcified neurocysticercosis lesions in patients with or without seizures. The subject's threshold of inflammation probably determines the occurrence of seizures. (Gupta RK, Awasthi R, Rathore RKS, et al. Understanding epileptogenesis in calcified neurocysticercosis with perfusion MRI. Neurology Feb 28, 2012;78:618-625). (Response and reprints. Prof Gupta: E-mail: rgupta@sgpgi.ac.in).

COMMENT. Albendazole and praziquantel, the two antiparasitic drugs used in treatment of neurocysticercosis, hasten radiologic resolution of cysts but may exacerbate the seizures resulting from the host inflammatory response. In patients with single inflamed brain parenchymal cysts, treatment is often withheld and is controversial. Approximately $10-20 \%$ of single cysticercal granuloma heal by calcification, and these patients are at higher risk of developing epilepsy (Rathore C et al. Editorial. Neurology 2012;78:612-613). Treatment is usually recommended for patients with nonenhancing or multiple cysticerci, and coadministration of corticosteroids for the first 2 or 3 days of therapy may decrease adverse effects. Arachnoiditis, vasculitis, or diffuse cerebral edema (cysticercal encephalitis) is treated with corticosteroids and albendazole or praziquantel. Anticonvulsant treatment is recommended until seizures are controlled for 1 to 2 years and there is radiological evidence of resolution. Calcified cysts usually require indefinite anticonvulsant therapy. Neurosurgery is indicated in patients with single intraventricular 